\title{
Mutaciones puntuales en los genes dhfr y dhps de Plasmodium falciparum de tres regiones endémicas para malaria en Colombia
}

\author{
John Alexander Galindo1, Fabio Aníbal Cristiano', Angélica Knudson², \\ Rubén Santiago Nicholls², Ángela Patricia Guerra ${ }^{1}$ \\ 1 Grupo de Bioquímica y Biología Celular, Instituto Nacional de Salud, Bogotá, D.C., Colombia
}

2 Grupo de Parasitología, Instituto Nacional de Salud, Bogotá, D.C., Colombia

Introducción. La acumulación progresiva de mutaciones en los genes dhfr y dhps lleva al parásito Plasmodium falciparum a evadir la acción de la sulfadoxina-pirimetamina, situación que aumenta el nivel de resistencia del parásito a estos medicamentos y conlleva a la aparición de fallas del tratamiento.

Objetivos. Determinar la frecuencia de mutaciones en los genes dhfry dhps de $P$. falciparum asociadas con resistencia a sulfadoxina-pirimetamina, en muestras de pacientes de tres zonas endémicas de Colombia: La Carpa, Guaviare; Casuarito, Vichada; Tierralta y Puerto Libertador, Córdoba.

Materiales y métodos. Se incluyeron 40 muestras de pacientes con malaria no complicada por $P$. falciparum. Los alelos 108, 59 y 164 del gen dhfr se analizaron mediante PCR específica de alelo y los alelos 51 del gen dhfr y 436, 437 y 540 del gen dhps por PCR y restricción enzimática.

Resultados. En el gen dhfr encontramos en todas las muestras las mutaciones asparagina 108 e isoleucina 51. No se detectaron alelos mutantes en los codones 59 y 164 del gen dhfr, ni en el codón 436 del gen dhps. La mutación glicina 437 estuvo presente en 36 muestras y el alelo silvestre alanina en tres de Tierralta y una de La Carpa. La mutación ácido glutámico 540 sólo se halló en Casuarito.

Conclusiones. En las poblaciones de $P$. falciparum analizadas prevalecen los alelos asparagina 108, isoleucina 51 y glicina 437, lo que indica un efecto acumulativo de mutaciones y la necesidad de vigilar la aparición de nuevos alelos mutantes que puedan conducir a la pérdida total de la eficacia de la sulfadoxina-pirimetamina.

Palabras clave: Plasmodium falciparum, sulfadoxina, pirimetamina, mutación, dihidrofolato reductasa, dihidropteroato sintasa, Colombia

Point mutations in dihydrofolate reductase and dihydropteroate synthase genes of Plasmodium falciparum from three endemic malaria regions in Colombia

Introduction. Plasmodium falciparum has the ability to counter the antiparasitic activity of sulphadoxinepyrimethamine by progressively accumulating mutations in the dihydrofolate reductase (dhfr) and dihydropteroate synthase (dhps) genes. These mutations gradually increase the resistance of the parasite to these drugs and lead to therapeutic failure.

Objectives. To determine the frequency of mutations associated with resistance to sulphadoxine and pyrimethamine in the $d h f r$ and $d h p s$ genes of $P$. falciparum in samples from patients in three endemic zones of Colombia -La Carpa, Guaviare; Casuarito, Vichada; and Tierralta and Puerto Libertador, Córdoba.

Materials and methods. Forty samples were selected from patients with uncomplicated $P$. falciparum malaria. The frequency profiles of the 108, 59 and 164 alleles of $d h f r$ were obtained by application of an allele-specific polymerase chain reaction, whereas the other alleles (alleles 51 of the dhfr gene and 436, 437 and 540 of $\mathrm{dhps}$ ) were obtained by polymerase chain reaction and restriction fragment length polymorphism.

Results. The $108 \mathrm{~N}$ and $51 \mathrm{I}$ mutations in the dhfr gene were found in all of the 40 samples. No mutant alleles were found in the 59 and 164 codons of the dhfrgene, or in the 436 codon of the dhps gene. The 437G mutation was observed in 36 samples and the wild-type allele was present in 3 from Tierralta and one from La Carpa. The 540E mutation was only detected in two samples from Casuarito.

Conclusions. The $108 \mathrm{~N}, 51 \mathrm{I}$ and $437 \mathrm{G}$ mutations prevail in the populations of $P$. falciparum, indicating a cumulative effect of mutations and the need to continue surveillance for other changes which can lead to the total loss of the efficacy of sulphadoxine-pyrimethamine.

Key words: Plasmodium falciparum, sulfadoxine, pyrimethamine, mutation, dihydrofolate reductase, dihydropteroate synthase, Colombia. 
La resistencia a los medicamentos antipalúdicos es uno de los principales factores que contribuyen al incremento de la enfermedad (1-4) y tiene implicaciones en el aumento de la mortalidad en zonas hiperendémicas y holoendémicas, y en el desarrollo y la expansión de focos de Plasmodium falciparum (5). Actualmente, el aumento progresivo de la resistencia a medicamentos es uno de los mayores retos que enfrenta el control de la malaria, situación que enfatiza la necesidad de ampliar los programas de vigilancia de la resistencia, mediante estudios de eficacia terapéutica, evaluación de la sensibilidad a medicamentos o el uso de marcadores moleculares que permitan de manera rápida y precisa la evaluación de la respuesta a los antipalúdicos (5).

El parásito $P$. falciparum es capaz de evadir la acción de los antifolatos mediante mutaciones puntuales que cambian ciertos aminoácidos de las enzimas dihidrofolato reductasa ( $d h f r)$-blanco de la pirimetamina-y dihidropteroato sintetasa (dhps) -sobre la que actúa la sulfadoxina- $(6,7)$, lo cual conlleva a una disminución de la afinidad de la enzima por el inhibidor.

El fenómeno de resistencia se debe en gran medida a la presión ejercida por los medicamentos, lo que ha permitido la selección de una serie de mutaciones sucesivas en los genes dhfr y dhps $(8,9)$. La primera mutación que aparece ocurre en el codón 108 de dhfr, esta mutación por sí sola es insuficiente para conferir resistencia in vivo a la pirimetamina (9). Posteriormente, y de manera independiente, pueden aparecer mutaciones en los codones 51 y 59, lo que aumenta el nivel de resistencia del parásito e incrementa el número de fallas del tratamiento. Una última mutación aparece en el codón 164 que, sumada a las mutaciones en los codones 108, 51 o 59, logra niveles de resistencia in vitro e in vivo que marcan el final del uso de la sulfadoxina-pirimetamina $(9,10)$.

Una vez se selecciona la mutación en el codon 108 del gen $d h f r$, se puede originar una serie de mutaciones en el gen dhps, de las cuales, la primera en aparecer es la mutación en el codón 437. Además, aparecen las mutaciones en los codones 540

\footnotetext{
Correspondencia:

Ángela Patricia Guerra, Grupo de Bioquímica y Biología Celular, Instituto Nacional de Salud, Bogotá, D.C., Colombia.

Telefax: 2200922

aguerra@ins.gov.co

Recibido: 17/03/09; aceptado:26/08/09
}

y 581 (9). En África se han descrito dos mutaciones en el codón 436; una de ellas (436P) se ha visto asociada con una mutación en el codón 613 y la otra (436A) raramente se encuentra cuando está presente la mutación en el codón 437 (10).

Las metodologías moleculares permiten detectar estas mutaciones y, según los estudios de Plowe (10), la presencia simultánea de alelos mutantes en los codones $108,51,59$ y 437 se asocia con la disminución de la sensibilidad a sulfadoxinapirimetamina, pero, una vez los codones silvestres 164 y 581 cambian al genotipo mutante, el parásito adquiere un mayor grado de resistencia que conlleva, generalmente, a la falla terapéutica.

Los estudios moleculares realizados en Colombia han analizado, básicamente, el codón 108 de dhfr (11-15) y han demostrado un notorio incremento de la mutación $108 \mathrm{~N}$, encontrándose una frecuencia de $51,6 \%$ en el trabajo más antiguo y de $100 \%$ en el más reciente. Poco se sabe con respecto a las otras mutaciones, ya que sólo se cuenta con el estudio de Schmider (12), quien reportó una frecuencia de $21 \%$ y $16 \%$ en las mutaciones 51 l y 59R del gen $d h f r$, respectivamente, y de $23 \%$ en la mutación 437G en el gen dhps.

En nuestro país, se han llevado a cabo varios estudios de eficacia de la monoterapia con sulfadoxina-pirimetamina en diferentes regiones endémicas y los resultados han mostrado un aumento en el número de fallas terapéuticas que va desde $15 \%$, promedio de los estudios hechos entre 1961 y 2003 (16), hasta 24\%, en estudios realizados entre 2000 y 2004 en la región de Urabá y el bajo Cauca (16), y 87,5\% en pacientes de la Amazonia (17). Sin embargo, en Colombia no se han llevado a cabo investigaciones tendientes a establecer asociaciones entre los patrones de mutaciones de los genes dhfr y dhps y la falla terapéutica.

Si bien desde finales de 2006 dejó de utilizarse la sulfadoxina-pirimetamina para el tratamiento de malaria no complicada por $P$. falciparum en Colombia, se consideró pertinente realizar un estudio molecular para establecer la frecuencia de las mutaciones asociadas con la resistencia a la sulfadoxina-pirimetamina en los genes dhfr y dhps, en tres regiones endémicas para malaria en Colombia, con muestras recolectadas entre los años 2002 y 2004, con el fin de conocer el efecto de la presión ejercida por sulfadoxina-pirimetamina durante casi dos décadas de uso sobre el genotipo de los parásitos circulantes en nuestro país. 
Los resultados obtenidos podrían servir de base para iniciar una vigilancia molecular que, eventualmente, permita reconsiderar la reintroducción de la sulfadoxina-pirimetamina en Colombia, en combinación con medicamentos derivados de la artemisinina.

\section{Materiales y métodos}

\section{Muestras de pacientes}

Se realizó un estudio descriptivo, en el cual se analizaron 40 muestras de pacientes con malaria no complicada infectados únicamente con $P$. falciparum, provenientes de cuatro poblaciones colombianas endémicas para malaria: 10 muestras procedían de La Carpa, corregimiento de San José del Guaviare (Guaviare), 10 de Casuarito, corregimiento de Puerto Carreño (Vichada), 10 de Tierralta y 10 de Puerto Libertador (Córdoba).

Se escogieron estas poblaciones por pertenecer a tres regiones ecoepidemiológicas diferentes de transmisión de $P$. falciparum en Colombia, las dos primeras en las regiones de la Amazonia y la Orinoquia, respectivamente, y las últimas en Urabá-bajo Cauca-alto Sinú.

El número de muestras procedentes de Vichada y Guaviare corresponde al total de muestras de $P$. falciparum que fue posible recolectar en un periodo de, aproximadamente, seis meses.

Con el fin de tener un número similar de muestras de las cuatro localidades, se decidió seleccionar aleatoriamente 10 muestras de Tierralta y Puerto Libertador. Todas las muestras de sangre fueron recolectadas sobre papel filtro durante los años 2002 a 2004. Además, se procesaron cuatro muestras de Casuarito, de pacientes que fueron tratados con amodiaquina más sulfadoxinapirimetamina, y a quienes se les hizo seguimiento clínico y parasitológico por parte de un médico en los días 1, 2, 3, 7 y 14, teniendo en cuenta que el día de inicio del tratamiento es el día 0.

El diagnóstico de malaria se hizo por gota gruesa y extendido de sangre periférica y la lectura fue hecha por microscopistas expertos. Posteriormente, el control de calidad se hizo en el Grupo de Parasitología del Instituto Nacional de Salud mediante una segunda evaluación.

Extracción de ADN y amplificación del gen dhfr mediante la reacción en cadena de la polimerasa

Para las muestras procedentes de La Carpa y Casuarito, el ADN se extrajo utilizando el estuche comercial (QIAmp DNA Micro kit QIAGEN) y siguiendo las recomendaciones del fabricante, y para las muestras de Tierralta y Puerto Libertador el ADN se extrajo mediante el método de Chelex y calentamiento (11).

Los alelos para la posición 108 del gen dhfr fueron analizados en un estudio previo mediante reacción en cadena de la polimerasa (PCR) específica de alelo (11); para determinar los alelos de los codones 51,59 y 164 del gen $d h f r$, se realizó una primera ronda de PCR (PCR universal) utilizando los iniciadores M1 y M7 (cuadro 1) en un volumen final de $20 \mu \mathrm{l}$ usando $300 \mu \mathrm{M}$ de dNTP, $0,25 \mu \mathrm{M}$ de cada iniciador, $1 \mathrm{U}$ de Taq ADN polimerasa (Invitrogen) y $1,5 \mathrm{mM}$ de $\mathrm{MgCl}_{2}$.

El programa de amplificación consistió en un ciclo inicial de desnaturalización a $94^{\circ} \mathrm{C}$ durante 3 minutos, 40 ciclos a $94^{\circ} \mathrm{C}$ durante un minuto, a $52^{\circ} \mathrm{C}$ durante 2 minutos y a $72^{\circ} \mathrm{C}$ durante un minuto, y una extensión final a $72^{\circ} \mathrm{C}$ durante 10 minutos (18). Los alotipos de los codones 59 y 164 se determinaron por PCR específica de alelo y el alotipo del codón 51, por restricción enzimática.

Se utilizaron como controles las cepas de referencia 3D7 (alelos silvestres) y Dd2 (alelos mutantes) para los codones 51 y 59 , y la cepa FCB1 (alelo silvestre) y V1/S (alelo mutante) para el codón 164 .

\section{Detección del alotipo en los codones 59 y 164 del gen dhfr}

Se realizó una segunda PCR utilizando los iniciadores específicos de alelo SP1 y FR-59W o SP1 y FR-59M, para definir el alelo silvestre o mutante en el codón 59 (cuadro 1); se hicieron algunas modificaciones a la metodología descrita por Chaparro (19) y las condiciones de la PCR fueron las siguientes: la reacción se llevó a un volumen final de $20 \mu$ usando $200 \mu \mathrm{M}$ de dNTP, 1 $\mu \mathrm{M}$ de cada iniciador, $1 \mathrm{U}$ de Taq ADN polimerasa (Invitrogen) y $1,5 \mathrm{mM}$ de $\mathrm{MgCl}_{2}$.

El programa de amplificación consistió en un ciclo inicial de desnaturalización a $95^{\circ} \mathrm{C}$ durante 3 minutos, 20 ciclos a $92^{\circ} \mathrm{C}$ durante 30 segundos, a $61^{\circ} \mathrm{C}$ durante 30 segundos y a $72^{\circ} \mathrm{C}$ durante 30 segundos, y una extensión final a $72^{\circ} \mathrm{C}$ durante 2 minutos.

Para detectar el alelo silvestre en el codón 164, se utilizaron los iniciadores SP1 y día13 y, para el alelo mutante, SP1 y día14 (cuadro 1); las condiciones de la PCR utilizada fueron similares a las descritas para el codón 59 y únicamente se 
Cuadro 1. Iniciadores usados en la amplificación de los genes dhfr y dhps. Iniciadores usados para amplificar el fragmento de interés de los genes dhfry dhps de $P$. falciparum.

\begin{tabular}{|c|c|c|c|c|}
\hline Amplicon & Alelo & \multicolumn{2}{|c|}{ Iniciador } & Secuencia $5^{\prime}-3^{\prime}$ \\
\hline \multicolumn{5}{|l|}{ Gen dhfr } \\
\hline \multirow[t]{2}{*}{ Universal $650 \mathrm{pb}$} & & $S$ & M1 & TTTATGATGGAACAAGTCTGC \\
\hline & & As & M7 & CTAGTATATACATCGCTAACA \\
\hline \multirow[t]{2}{*}{ Anidada $594 \mathrm{pb}$} & & $S$ & M3b & TGATGGAACAAGTCTGCGACGTT \\
\hline & & As & M9 & CTGGAAAAAATACATCACATTCATATG \\
\hline \multicolumn{2}{|l|}{ Específico de alelo } & $S$ & SP1 & ATGATGGAACAAGTCTGCGAC \\
\hline \multirow[t]{2}{*}{ Codón 59190 pb } & C59 & As & FR-59W & ATGTTGTAACTGCACA \\
\hline & $59 R$ & As & FR-59M & ATGTTGTAACTGCACG \\
\hline \multirow[t]{2}{*}{ Codón 164505 pb } & $\mid 164$ & As & Día 13 & CAACGGAACCTCCTAT \\
\hline & $164 \mathrm{~L}$ & As & Día 14 & CAACGGAACCTCCTAA \\
\hline \multicolumn{5}{|l|}{ Gen dhps } \\
\hline \multirow[t]{2}{*}{ Universal 770 pb } & & $S$ & N1 & GATTCTTTTTCAGATGGAGG \\
\hline & & As & N2 & TTCCTCATGTAATTCATCTGA \\
\hline \multirow[t]{2}{*}{ Anidada $711 \mathrm{pb}$} & & $S$ & $\mathrm{R} 2$ & AACCTAAACGTGCTGTTCAA \\
\hline & & As & $\mathrm{R}$ & AATTGTGTGATTTGTCCACAA \\
\hline
\end{tabular}

Amplicon: producto que amplifican los iniciadores; S: iniciador sentido; As: iniciador antisentido.

modificó la temperatura de alineamiento de los iniciadores a $62^{\circ} \mathrm{C}$ y se redujo el número de ciclos de amplificación a 15.

\section{Detección del alotipo en el codón 51 del gen dhfr por PCR y restricción enzimática}

A partir de los productos amplificados en la primera ronda de PCR, se realizó una segunda ronda (PCR anidada) en la que se utilizaron los iniciadores M3b y M9 (cuadro 1) con las mismas condiciones descritas para la universal.

El programa de amplificación consistió en un ciclo inicial de desnaturalización a $94^{\circ} \mathrm{C}$ durante 3 minutos, 4 ciclos a $94^{\circ} \mathrm{C}$ durante un minuto, a $44^{\circ} \mathrm{C}$ durante 2 minutos y a $72^{\circ} \mathrm{C}$ durante un minuto, y posteriormente, 34 ciclos a $94^{\circ} \mathrm{C}$ durante un minuto, a $44^{\circ} \mathrm{C}$ durante un minuto y a $72^{\circ} \mathrm{C}$ durante un minuto, y una extensión final a $72^{\circ} \mathrm{C}$ durante 10 minutos (18). El producto obtenido en esta reacción (594 pb) fue sometido a digestión enzimática con la enzima Tsp509 I (20). El patrón de restricción que se obtuvo para la cepa que presenta el alelo silvestre (3D7) fue: 149, 120, 66, 65, 64, 54, 46, 15, $10,5 \mathrm{pb}$ y el patrón para la cepa con el alelo mutante (Dd2) es: 213, 120, 66, 65, 54, 46, 15, 10, 5 pb.

\section{Amplificación por PCR del gen dhps y restricción enzimática}

Para determinar los alelos de los codones 436, 437 y 540 del gen dhps se realizó una primera ronda de PCR (PCR universal) utilizando los iniciadores N1 y N2 (cuadro 1) con las mismas concentraciones de reactivos descritas para la PCR universal del gen dhfr y el mismo programa de amplificación, modificando únicamente la temperatura de alineamiento de los iniciadores que fue de $52^{\circ} \mathrm{C}$ (18).

El producto obtenido en esta reacción (711 pb) fue analizado por restricción enzimática utilizando las enzimas MSPA1 I que detectan el alelo mutante 436A, Ava II que detecta el alelo mutante 437G y Fok I que detecta el alelo mutante $540 \mathrm{E}(10,21)$. Como controles se utilizaron las cepas T9-96, 3D7, HB3, T9-94 e IEC513/86. La cepa T9-96 presenta el genotipo mutante 436A, y la cepa 3D7 el genotipo silvestre S436, y cuando T9-96 es tratada con la enzima MSPA1 I se produce un corte generando dos fragmentos, uno de $633 \mathrm{pb}$ y otro de $78 \mathrm{pb}$. En el caso de 3D7 no se produce ningún corte. La cepa 3D7 presenta el genotipo mutante 437G, y cuando es tratada con la enzima AVA II se produce un corte generando dos fragmentos, uno de 630 pb y otro de $81 \mathrm{pb}$. La cepa HB3 o T9-94 presenta el genotipo silvestre A437 y no hay corte con la enzima. La cepa IEC513/86 presenta el genotipo mutante $540 \mathrm{E}$, hay corte con la enzima Fok I y se producen dos fragmentos, uno de $390 \mathrm{pb}$ y otro de $321 \mathrm{pb}$. La cepa 3D7 o HB3 presenta el genotipo silvestre K540 y no hay corte con la enzima.

A partir de los productos amplificados en la primera ronda de PCR se realizó una PCR anidada en la que se utilizaron los iniciadores $R 2$ y $R$ (cuadro 1 ) con las mismas condiciones descritas para la PCR universal.

Enzima Fok I, la cepa IEC513/86 presenta el genotipo mutante $540 \mathrm{E}$, hay corte con la enzima y 
se producen dos fragmentos, uno de $390 \mathrm{pb}$ y otro de $321 \mathrm{pb}$. La cepa 3D7 o HB3 presenta el genotipo silvestre K540 y no hay corte con la enzima.

Todos los productos de PCR y los productos digeridos con las enzimas de restricción fueron resueltos en geles de agarosa al $2 \%$, teñidos en solución acuosa de bromuro de etidio $1 \mu \mathrm{g} / \mathrm{ml}$, visualizados sobre luz ultravioleta y analizados por el programa One-Dscan one-dimensional electrophoresis ${ }^{\circledR}$ (Scanalytic Fairfax, VA).

\section{Análisis de datos}

Se determinó la frecuencia de cada alelo en los 7 codones estudiados y se determinaron los patrones de mutaciones (doble, triple y cuádruple) y su distribución por localidades.

El análisis descriptivo de las variables se efectuó con las herramientas numéricas convencionales (proporciones, medias, medianas, medidas de dispersión), dependiendo de las características de cada variable. Los estimadores fueron calculados junto con sus respectivos intervalos de confianza del $95 \%$. Los anteriores procedimientos fueron ejecutados con el programa Stata $8 \AA$.

\section{Aspectos éticos}

El estudio fue aprobado por el Comité de Ética del Instituto Nacional de Salud. De acuerdo con la Resolución № 008430 de 1993 del Ministerio de Salud, República de Colombia, este estudio se clasificó como de riesgo mínimo. De todos los pacientes se solicitó y obtuvo consentimiento informado escrito y se les administró tratamiento antipalúdico.

\section{Resultados}

Al analizar el gen dhfr se encontró que en los codones 108 y 51, las 40 muestras (100\%) presentaron los alelos mutantes $108 \mathrm{~N}$ y $51 \mathrm{l}$ (figura $1 \mathrm{~A})$, mientras que para los codones 59 y 164 toda la población estudiada presentó los alelos silvestres C59 e I164 (figura 1B).

Al analizar el gen dhps se encontró que, para el codón $437,90 \%$ de las muestras (36/40) presentó el alelo mutante $437 \mathrm{G}$ y 4 muestras tuvieron el alelo silvestre A437, 3 de Tierralta $(7,5 \%)$ y una de La Carpa $(2,5 \%)$. No se detectó ninguna muestra con alelo mutante en el codón 436 y solamente se encontraron dos muestras con la mutación 540E (figura 1C), las cuales procedían de Casuarito.

Los resultados de ambos genes se evaluaron en conjunto y se determinó la frecuencia de mutantes dobles, triples y cuádruples; así, todas las muestras analizadas presentaron, al menos, dos mutaciones (108N y $51 \mathrm{I}), 90 \%$ presentó una tercera mutación (437G) y 5\% presentó una cuarta mutación (540E).

De los cuatro pacientes de Casuarito que fueron tratados con amodiaquina $(25 \mathrm{mg})$ más sulfadoxina $(1.500 \mathrm{mg})$ y pirimetamina $(75 \mathrm{mg})$, tres presentaron respuesta clínica adecuada y uno, falla terapéutica precoz. Este paciente exhibió un patrón de mutante cuádruple (108N, 51I, 437G y 540E) a diferencia de los pacientes con respuesta clínica adecuada, quienes mantuvieron parásitos con patrón de mutante triple (108N, 51 l y $437 \mathrm{G}$ ).

En la figura 2 se puede apreciar la distribución de mutantes triples y cuádruples por localidad.

\section{Discusión}

Las muestras que presentaron el patrón de mutante cuádruple (108N, 51I, 437G y 540E) sólo se encontraron en Casuarito, localidad ubicada sobre la ribera occidental del río Orinoco al frente de Puerto Ayacucho (Venezuela), en una zona que ecológicamente corresponde a bosque seco tropical. La mayor parte de su territorio posee características que permiten clasificarla como zona de riesgo para transmisión de malaria; así, en el año 2001, el total de casos de malaria reportados en el departamento de Vichada fue de 109 con un índice parasitario anual (IPA) de 6,85; en el 2.002, 174 con un IPA de 10,61 y, en el 2003, 314 con un IPA de 18,6.

Una posible explicación para el hallazgo del mutante cuádruple es que los parásitos analizados proceden del vecino país como consecuencia de las migraciones de la población o el intercambio socioeconómico propio de las zonas fronterizas, o debido a la ubicación de Casuarito, localidad donde losparásitos posiblementehanestado expuestospor más tiempo a sulfadoxina-pirimetamina comparados con parásitos procedentes de Tierralta, Puerto Libertador o La Carpa. En Venezuela, la sulfadoxinapirimetamina fue el esquema terapéutico de primera línea para la malaria no complicada por $P$. falciparum por más de 20 veinte años (hasta el 2002); esto sugiere que los parásitos circulantes en ese país estuvieron bajo influencia y presión de selección con sulfadoxina-pirimetamina por más tiempo, situación que conllevó a la selección de mutaciones en los genes dhfr y dhps de $P$. falciparum (22).

Urdaneta et al. (21) realizaron un estudio molecular con muestras de pacientes recolectadas en 1995 en 
A

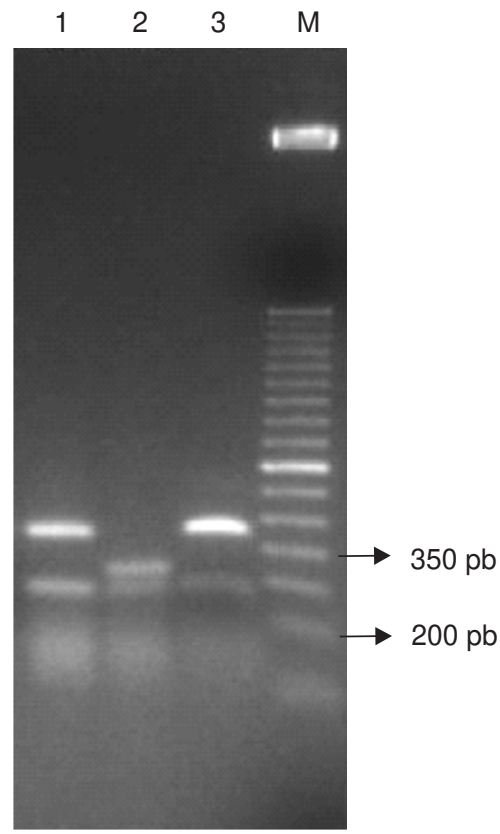

C

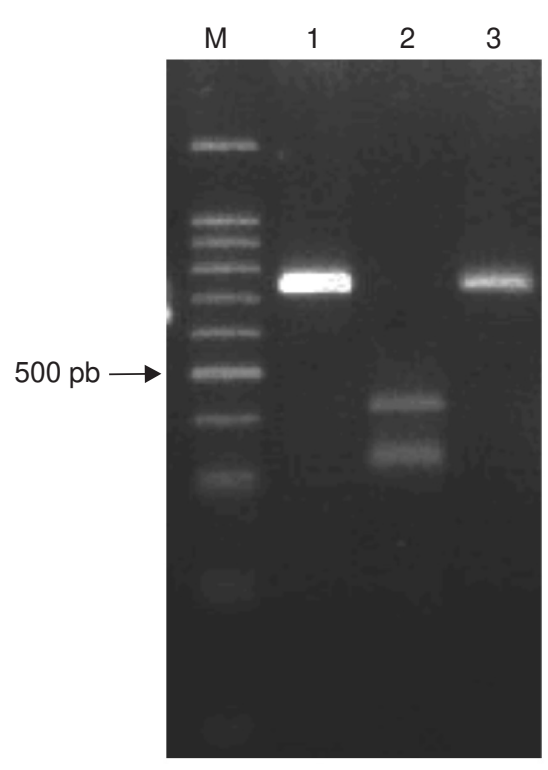

B

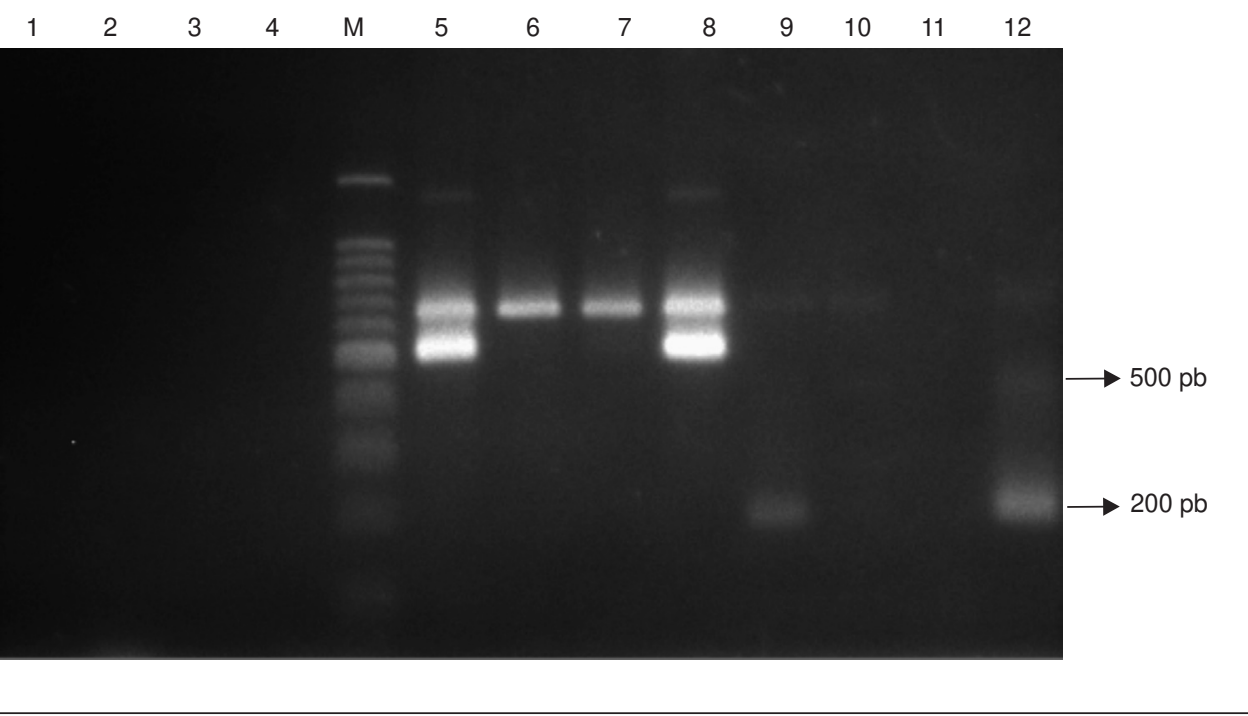

Figura 1A. Digestión con la enzima de restricción Tsp509 I para detección del alelo silvestre en el codón 51 del gen dhfr. Gel de agarosa al $2 \%$ teñido con bromuro de etidio. Carril 1 y 3: ADN de cepa de referencia Dd2 (alelo mutante). Carril 2: ADN de cepa de referencia 3D7 (alelo silvestre). M: marcador de peso molecular de $50 \mathrm{pb}$.

Figura 1B. Amplificación por PCR usando iniciadores alelo específicos para detectar los alelos presentes en los codones 59 y 164 del gen $d h f r$. Gel de agarosa al 2\% teñido con bromuro de etidio. Carril 1 al 4: controles negativos para reacción con iniciadores SP1 y FR-59W, SP1 y FR-59M, SP1 y día 13, y SP1 y día 14. M: marcador de peso molecular de 100 pb. Carril 5 y 6: Iniciadores SP1 y día 13 con ADN de cepa control FCB-1 y V1/S, respectivamente. Carril 7 y 8: Iniciadores SP1 y día 14 con ADN de cepa control FCB-1 y V1/S, respectivamente. Carril 9 y 10: Iniciadores SP1 y FR-59W con ADN de cepa control 3D7 y Dd2, respectivamente. Carril 9 y 10: Iniciadores SP1 y FR-59M con ADN de cepa control 3D7 y Dd2, respectivamente.

Figura 1C. Digestión con la enzima de restricción Fok I para detección del alelo mutante en el codón 540 del gen dhps. Gel de agarosa al 1\% teñido con bromuro de etidio. M: marcador de peso molecular de $100 \mathrm{pb}$. Carril 1: ADN de cepa de referencia 3D7 (alelo silvestre). Carril 2: ADN de cepa de referencia IEC513/86 (alelo mutante). Carril 3: muestra de Tierralta con alelo silvestre. 


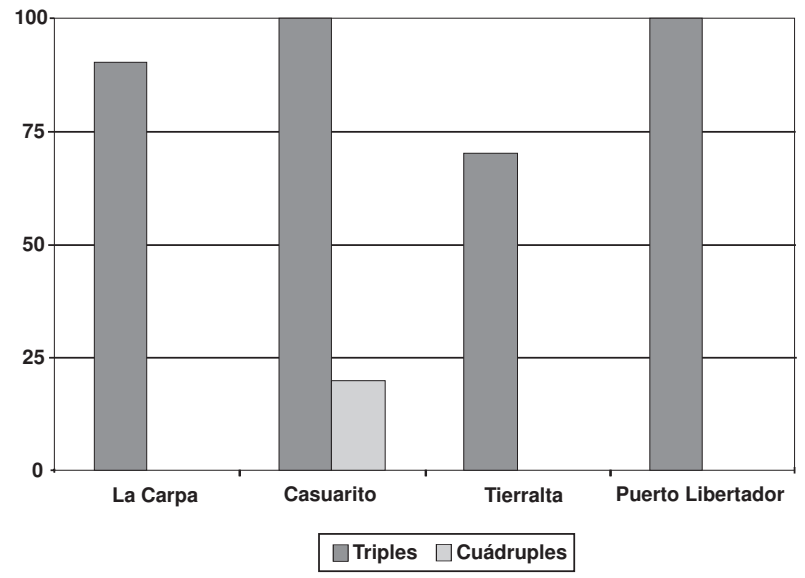

Figura 2. Distribución de mutantes triples y cuádruples por localidad. Mutantes triples: muestras con las mutaciones 108N, $51 \mathrm{l}$ (dhfr) y 437G (dhps); mutantes cuádruples: muestras con las mutaciones $108 \mathrm{~N}, 51 \mathrm{l}$ (dhfr), 437G y 540E (dhps). En la localidad de Casuarito las 10 muestras analizadas presentaron el patrón de mutante triple y, entre éstas, sólo dos exhibieron adicionalmente la mutación 540E.

el estado de Bolívar, zona que informó altos niveles de falla terapéutica a sulfadoxina-pirimetamina. Los resultados mostraron la presencia de las mutaciones $108 \mathrm{~N}, 50 \mathrm{R}$ e $51 \mathrm{l}$ en el gen dhfr con frecuencias de $96 \%, 64 \%$ y $96 \%$, respectivamente, y las mutaciones $437 \mathrm{G}$ y $581 \mathrm{G}$ en el gen dhps con frecuencias del $100 \%$ y $96 \%$, respectivamente (21). Por otra parte, Contreras et al. (23) encontraron la misma tendencia de mutaciones, lo cual sugiere que los parásitos mantienen en su genoma estas mutaciones desde antes del 2000.

En este estudio no se detectó el genotipo mutante en el codón 59 del gen dhfr en ninguno de los pacientes de las tres regiones analizadas, resultado que está de acuerdo con el trabajo de Méndez (24), realizado con pacientes procedentes de Buenaventura (Costa Pacífica), y con varios reportes de diferentes países suramericanos $(8,21$ 23,25-27). Lo anterior sugiere que el codón 59 no es afectado por la presión ejercida con la sulfadoxinapirimetamina y que el mecanismo de selección de mutaciones puede ser distinto en parásitos de Suramérica comparado con el de parásitos de África $(28,29)$, pero esto debe ser corroborado con estudios de parásitos provenientes de diferentes regiones del país y de otros países, así como con muestras tomadas en diferentes periodos.

Por lo tanto, de acuerdo con la información disponible hasta la fecha, la determinación del alelo presente en este codón no parece brindar información relevante en estudios colombianos.
La alta frecuencia del triple mutante $(108 \mathrm{~N}, 51 \mathrm{l}$ y $437 \mathrm{G}$ ) indica que hay un efecto acumulativo de mutaciones, las cuales se asocian con disminución de la sensibilidad a la sulfadoxina-pirimetamina y a una posible fijación de estos alelos en el genoma de las poblaciones del parásito analizadas $(8,9,22)$. Esta disminución de la sensibilidad asociada con las tres mutaciones no necesariamente implica falla del tratamiento, sino que puede reflejarse en un tiempo más prolongado de eliminación del parásito (24).

Por otro lado, también se ha visto que las infecciones conparásitosqueexhiben las mutaciones $108 \mathrm{Ny} 511$, presentan una mayor densidad de formas sexuales en sangre, lo cual puede darse por un aumento del tiempo de supervivencia del parásito que está bajo la presión del medicamento, permitiéndole a los estadios asexuales diferenciarse en gametocitos y favoreciendo su rápida diseminación (24).

En Tierralta se encontró el mutante triple $(108 \mathrm{~N}$, 51 l y $437 \mathrm{G}$ ) en $70 \%$ de las muestras y, aunque estos resultados no provienen de un estudio de eficacia de sulfadoxina-pirimetamina que nos permita establecer asociaciones entre los patrones de mutaciones múltiples y la falla al tratamiento, vale la pena tener en cuenta los resultados de un estudio de eficacia de la combinación amodiaquina más sulfadoxina-pirimetamina realizado en 2006 por el Instituto Nacional de Salud, en pacientes procedentes del municipio de Tierralta (30), en el cual se encontró una eficacia de 94,3\% (50/53). Lo anterior sugiere que, aunque el mutante triple se encuentra ampliamente distribuido en Córdoba, esta combinación de mutaciones no es suficiente para anular el efecto antipalúdico de la sulfadoxinapirimetamina o que, en caso de que ello ocurra, la amodiaquina logra asegurar la curación parasitológica, lo que confirma que, aun cuando haya presencia de mutaciones, la administración de terapia combinada con amodiaquina todavía es exitosa y puede ser de utilidad en el país.

Aunque no se analizó el codón 581 del gen dhps, todas las muestras presentaron el alelo silvestre en el codón 164 de $d h f r$, situación que sugiere que en estas localidades aún es posible usar sulfadoxinapirimetamina, ya que la aparición de estas dos mutaciones se asocia con la fase final del desarrollo de resistencia a sulfadoxina-pirimetamina (9).

Por otro lado, la diferencia en el análisis molecular entre el paciente con falla terapéutica precoz y los tres pacientes con respuesta clínica adecuada radicó en la aparición de la mutación $540 E$, lo que sugiere que las mutaciones que ofrecerían mayor 
información en el momento actual serían en las posiciones 540, 581 y 164, siendo esta última la que determina el final de la vida útil de la sulfadoxinapirimetamina (9).

Es de gran utilidad evaluar las posiciones 540 y 581 en zonas con alta eficacia de la sulfadoxinapirimetamina, ya que estos resultados brindarían información sobre el orden de aparición de las mutaciones y ayudarían a definir cuál de éstas tiene mayor asociación con la falla terapéutica. Teniendo en cuenta que las poblaciones de parásitos que circulan en estas tres regiones, en general, poseen el triple mutante, proponemos que el análisis de los codones 540, 581 y 164 es suficiente para dar información sobre el nivel de resistencia a sulfadoxina-pirimetamina que compromete la respuesta clínica del paciente en Colombia.

A partir del segundo semestre de 2006 se implementó en Colombia el uso de combinaciones terapéuticas con derivados de artemisinina para el tratamiento de la malaria no complicada por $P$. falciparum. Aunque ninguna de las combinaciones actualmente en uso en Colombia incluye la sulfadoxina-pirimetamina, sería conveniente continuar con la vigilancia molecular de la resistencia a sulfadoxina-pirimetamina, puesto que se espera que, como consecuencia de la suspensión del uso de este medicamento, las poblaciones de parásitos silvestres aumenten gradualmente, y se pueda considerar como una alternativa el uso de combinaciones de sulfadoxina-pirimetamina, con derivados de artemisinina. Esto podría realizarse mediante la implementación de una red de vigilancia molecular, con énfasis en regiones de difícil acceso y donde hacer el seguimiento de los pacientes es casi imposible.

\section{Agradecimientos}

Los autores agradecen la colaboración recibida de las siguientes instituciones para la realización del trabajo de campo: Secretaría Departamental de Salud de Córboba, Secretaría Municipal de Salud de Tierralta, Secretaría Municipal de Salud de Puerto Libertador, microscopistas de la empresa asociativa de trabajo DIFUSANJOR, Secretaría Departamental de Salud de Guaviare, Secretaría Departamental de Salud de Vichada, Laboratorio Departamental de Salud Pública de Vichada y Puesto de Salud de Casuarito.

\section{Conflicto de intereses}

Los autores manifestamos expresamente que durante la realización del presente trabajo no existió conflicto de interés alguno que pudiera haber afectado los resultados obtenidos.

\section{Financiación}

Este estudio fue cofinanciado con recursos del Fondo de Investigaciones en Salud, Ministerio de la Protección Social-Colciencias, Proyecto Código 2104-04-11813, Contrato No. RC 134-2002 y del Instituto Nacional de Salud. John Alexander Galindo estuvo vinculado a este proyecto gracias al Programa Jóvenes Investigadores e Innovadores de Colciencias, convenio interadministrativo especial de cooperación No. 44-2005 Colciencias-INS y el contrato de prestación de servicios profesionales No. 150-2005.

\section{Referencias}

1. Le Bras J, Durand R. The mechanisms of resistance to antimalarial drugs in Plasmodium falciparum. Fundam Clin Pharmacol. 2003;17:147-53.

2. Wyler DJ. Malaria-resurgence, resistance, and research (first of two parts). N Engl J Med. 1983;308:875-8.

3. Hyde JE. Mechanisms of resistance of Plasmodium falciparum to antimalarial drugs. Microbes Infect. 2002;4:16574.

4. Sibley CH, Hyde JE, Sims PF, Plowe CV, Kublin JG, Mberu EK, et al. Pyrimethamine-sulfadoxine resistance in Plasmodium falciparum: what next? Trends Parasitol. 2001;17:582-8

5. Noeld $\mathbf{H}$, Wongsrichanalai $\mathrm{C}$, Wernsdorfer WH. Malaria drug-sensitivity testing: new assays, new perspectives. Trends Parasitol. 2003;19:175-81.

6. Gatton ML, Martin LB, Cheng Q. Evolution of resistance to sulfadoxine-pyrimethamine in Plasmodium falciparum. Antimicrob Agents Chemother. 2004;48:2116-23.

7. Yuthavong Y. Basis for antifolate action and resistance in malaria. Microbes Infect. 2002;4:175-82.

8. Sharma YD. Genetic alteration in drug resistance markers of Plasmodium falciparum. Indian J Med Res. 2005;121:13-22.

9. Cortese JF, Caraballo A, Contreras CE, Plowe CV. Origin and dissemination of Plasmodium falciparum drug-resistance mutations in South America. J Infect Dis. 2002;186:999-1006.

10. Plowe CV, Cortese JF, Djimde A, Nwanyanwu OC, Watkins WM, Winstanley PA, et al. Mutations in Plasmodium falciparum dihydrofolate reductase and dihydropteroate synthase and epidemiologic patterns of pyrimethamine-sulfadoxine use and resistance. $J$ Infect Dis. 1997;176:1590-6.

11. Guerra AP, Knudson A, Nicholls RS, Galindo JA, Ravid Z, Rahirant S, et al. Genotipificación de los genes msp1 (bloque 2) y dhfr (codón108) de Plasmodium falciparum en muestras de campo recolectadas en cuatro localidades endémicas de Colombia. Biomédica. 2006;26:101-12.

12. Schmider N, Peyerl-Hoffmann G, Restrepo M, Jelinek T. Short communication: point mutations in the dihydrofolate 
reductase and dihydropteroate synthase genes of Plasmodium falciparum isolates from Colombia. Trop Med Int Health. 2003;8:129-32.

13. Gómez D, Chaparro J, Rubiano C, Rojas MO, Wasserman M. Genetic diversity of Plasmodium falciparum field samples from an isolated Colombian village. Am J Trop Med Hyg. 2002;67:611-6.

14. Rallón NI, Osorio LE, Giraldo LE. Lack of an association between the Asn-108 mutation in the dihydrofolate reductase gene and in vivo resistance to sulfadoxine/ pyrimethamine in Plasmodium falciparum. Am J Trop Med Hyg. 1999;61:245-8.

15. Giraldo LE, Acosta MC, Labrada LA, Praba A, Montenegro-James S, Saravia NG, et al. Frequency of the Asn-108 and Thr-108 point mutations in the dihydrofolate reductase gene in Plasmodium falciparum from southwest Colombia. Am J Trop Med Hyg. 1998;59:124-8.

16. Blair S, Carmona-Fonseca J, Piñeros JG, Ríos A, Álvarez $\mathrm{T}$, Álvarez G, et al. Therapeutic efficacy test in malaria falciparum in Antioquia, Colombia. Malar J. 2006;5:14.

17. Osorio L, Pérez LP, González IJ. Evaluación de la eficacia de los medicamentos antimaláricos en Tarapacá, Amazonas colombiano. Biomédica. 2007;27:133-40.

18. Pearce RJ, Drakeley C, Chandramohan D, Mosha F. Molecular determination of point mutation haplotypes in the dihydrofolate reductase and dihydropteroate synthase of Plasmodium falciparum in three districts of Northern Tanzania. Antimicrob Agents Chemother. 2003;47:1347-54.

19. Chaparro J, Wasserman M. Comparación de técnicas in vitro para detectar resistencia de Plasmodium falciparum a medicamentos. Biomédica. 1999;19:103-14.

20. Duraisingh MT, Curtis J, Warhurst DC. Plasmodium falciparum: detection of polymorphisms in the dihydrofolate reductase and dihydropteroate synthetase genes by PCR and restriction digestion. Exp Parasitol. 1998;89:1-8.

21. Urdaneta L, Plowe C, Goldman I, Lal A. Point mutations in dihydrofolate reductase and dihydropteroate synthase genes of Plasmodium falciparum isolates from Venezuela. Am J Trop Med Hyg. 1999;61:457-62.

22. McCollum AM, Mueller K, Villegas L, Udhayakumar V, Escalante AA. Common origin and fixation of Plasmodium falciparum dhfr and dhps mutations associated with sulfadoxine-pyrimethamine resistance in a low-transmission area in South America. Antimicrob Agents Chemother. 2007;51:2085-91.

23. Contreras CE, Cortese JF, Caraballo A, Plowe CV. Genetics of drug-resistant Plasmodium falciparum malaria in the Venezuelan state of Bolívar. Am J Trop Med Hyg. 2002;67:400-5.

24. Mendez F, Muñoz A, Carrasquilla G, Jurado D, ArévaloHerrera M, Cortese J, et al. Determinants of treatment response to sulfadoxine-pyrimethamine and subsequent transmission potential in falciparum malaria. Am J Epidemiol. 2002;156:230-8.

25. Zhou Z, Griffing SM, de Oliveira AM, McCollum AM, Quezada WM, Arrospide N, et al. Decline in sulfadoxinepyrimethamine-resistant alleles after change in drug policy in the Amazon region of Peru. Antimicrob Agents Chemother. 2008;52:739-41.

26. Peek R, van Gool T, Panchoe D, Greve S, Bus E, Resida L. Drug resistance and genetic diversity of Plasmodium falciparum parasites from Suriname. Am J Trop Med Hyg. 2005;73:833-8.

27. Vasconcelos KF, Plowe CV, Fontes CJ, Kyle D, Wirth DF, Pereira da Silva LH, et al. Mutations in Plasmodium falciparum dihydrofolate reductase and dihydropteroate synthase of isolates from the Amazon region of Brazil. Mem Inst Oswaldo Cruz. 2000;95:721-8.

28. Talisuna A, Langi P, Mutabingwa T, Watkins W, Van Marck E, Egwang T, et al. Population-based validation of dihydrofolate reductase gene mutations for the prediction of sulfadoxine-pyrimethamine resistance in Uganda. Trans $R$ Soc Trop Med Hyg. 2003;97:338-42.

29. Kublin JG, Dzinjalamala FK, Kamwendo DD, Malkin EM, Cortese JF, Martino LM, et al. Molecular markers for failure of sulfadoxine-pyrimethamine and chlorproguanil-dapsone treatment of Plasmodium falciparum malaria. J Infect Dis. 2002;185:380-8.

30. Pérez MA, Cortés LJ, Guerra AP, Knudson A, Usta C, Nicholls RS. Eficacia de la combinación amodiaquina más sulfadoxina/pirimetamina y de la cloroquina para el tratamiento del paludismo en Córdoba, Colombia, 2006. Biomédica. 2008;28:148-59. 